\title{
Hemocompatibility study of a bacterial cellulose/polyvinyl alcohol nanocomposite
}

\author{
Alexandre F. Leitão ${ }^{a}$, Swati Gupta ${ }^{\mathrm{b}, \mathrm{c}}$, João Pedro Silva ${ }^{\mathrm{a}}$, Ilya Reviakine ${ }^{\mathrm{b}, \mathrm{d}}$, Miguel Gama ${ }^{\mathrm{a}, *}$ \\ ${ }^{a}$ CEB - Centre of Biological Engineering, IBB - Institute for Biotechnology and Bioengineering, Department of Biological Engineering, University of Minho, \\ Campus de Gualtar, 4710-057 Braga, Portugal \\ ${ }^{\mathrm{b}}$ Centre for Cooperative Research in Biomaterials - CIC biomaGUNE, Paseo Miramón 182, 20009 San Sebastián, Gipuzkoa, Spain \\ ${ }^{\mathrm{c}}$ Department of Biochemistry and Molecular Biology, University of the Basque Country, Leioa 48940, Spain \\ d Bilbao International School of Engineering, University of the Basque Country, Alameda Urquijo S-N, Bilbao 48013, Spain
}

\section{A R T I C L E I N F O}

\section{Article history:}

Received 4 January 2013

Received in revised form 14 June 2013

Accepted 17 June 2013

\section{Keywords:}

Bacterial cellulose

Biocompatibility

Biomaterials

Polyvinyl alcohol

Platelets

Hemocompatibility

\begin{abstract}
A B S T R A C T
Bacterial cellulose (BC) has been suggested to be a suitable biomaterial for the development of cardiovascular grafts. The combination of $\mathrm{BC}$ with polyvinyl alcohol (PVA) results in nanocomposites with improved properties. Surprisingly, there are very few studies on the $\mathrm{BC}$-blood interaction. This is the focus of this paper. We present the first thorough assessment of the hemocompatibility of the BC/PVA nanocomposite. Whole blood clotting time, plasma recalcification, Factor XII activation, platelet adhesion and activation, hemolytic index and complement activation are all determined. The platelet activation profiles on $\mathrm{BC}$ and BC/PVA surfaces are comprehensively characterized. BC and BC/PVA outperformed ePTFE - used as a point of comparison - thus evidencing their suitability for cardiovascular applications.
\end{abstract}

(c) 2013 Elsevier B.V. All rights reserved.

\section{Introduction}

With the increase in the prevalence of cardiovascular diseases in the adult population, cardiovascular surgeries are becoming more and more common in operating rooms across the world. Among the most prevalent cardiovascular diseases is atherosclerosis which is generally treated by performing a bypass surgery. The procedure requires the application of a biological or a synthetic graft that will allow the blood flow to be redirected around an occluded portion of the vessel. Biological grafts deteriorate over time due to the natural progression of the patients' disease or may be unavailable [1]. Therefore, synthetic grafts are used. These are commonly made of polyethylene terephthalate (PET, commercially known as Dacron) or expanded polytetrafluoroethylene (ePTFE). However, they do not perform well in medium or small-caliber vessels such as coronary arteries [1]. In larger-caliber vessels characterized by high shear rates these materials do provide good

\footnotetext{
* Corresponding author at: Department of Biological Engineering, University of Minho, Campus de Gualtar, 4710-057 Braga, Portugal. Tel.: +351 253604 418; fax: +351253604 429.

E-mail addresses: afleitao@deb.uminho.pt(A.F.Leitão),sgupta@cicbiomagune.es (S. Gupta),jpsilva@deb.uminho.pt (J.P. Silva), ireviakine@cicbiomagune.es (I. Reviakine), fmgama@deb.uminho.pt (M. Gama).
}

mechanical compliance. However, they have a strong affinity for proteins, which adsorb on the surface of the material. This results in a layer, the pseudo-intima, which promotes platelet adhesion and activation and consequent clot formation $[2,3]$. In low caliber vessels the protein build-up on the luminal surface and platelet activity ultimately lead to thrombosis. There is therefore a strong need for new materials for the construction of vascular grafts for small-caliber vessel applications, and a large amount of work has been aimed at finding natural alternatives to the synthetic polymer materials for making grafts.

In order to be suitable for this application, the material has to be hemocompatible, i.e., it must be able to remain in contact with blood without inducing toxicity or activating the intrinsic and extrinsic coagulation cascades or the complement system [4]. By triggering these blood reactions, foreign materials cause thrombosis and inflammation at the graft site or embolism. Emboli that occur due to the detachment of thrombi from the graft site or their formation downstream of the implant are the main cause of synthetic grafts failure [2].

One promising candidate is bacterial cellulose (BC). This polymer is a highly pure linear polysaccharide, consisting of $\beta$ $(1 \rightarrow 4)$-linked D-glucose monomers. It is secreted by bacteria of the Gluconacetobacter genus. Once secreted, it forms a fibrous network hydrogel. BC has been extensively studied for biomedical applications due to its morphology, high purity, water-holding capacity, 
tensile strength, malleability and biocompatibility [5-7] and has been proposed for applications such as wound dressings [8], artificial skin [9] or blood vessels [6,10-12] and as a scaffold for tissue engineering [7,13-15].

Polyvinyl alcohol (PVA) has also been studied for potential biomedical applications. Its uncomplicated structure allows crosslinking of the polymer chains by either chemical agents or by physical crosslinking through thermal cycling [16]. The hydrogels formed by PVA generally present good tensile strength, flexibility and elasticity [17], allied with high water retention capability, non-toxicity and non-carcinogenicity. PVA has been studied for applications in tissue reconstruction and replacements, drug delivery, cardiovascular stents and wound covering bandages, among others [16,18].

$\mathrm{BC}$ has high tensile strength but somewhat limited elasticity. On the other hand, PVA is highly elastic but its tensile strength is limited. A compromise would be ideal for potential applications in cardiovascular surgery: sufficient tensile strength allied with enough elasticity to allow for greater mechanical compliance. The BC/PVA nanocomposites indeed meet these criteria, as we and others reported $[16,19,20]$. We described previously one such nanocomposite, consisting of an interpenetrated polymer network of PVA fibers entangled inside the BC matrix, bridging gaps and linking individual $B C$ fibers. The mechanical properties of the BC/PVA nanocomposites can be adjusted by thermal cycling. This allows tailoring of the nanocomposite mechanical properties to better mimic those of the arterial vessel walls.

While the potential of $\mathrm{BC}$ as a biomaterial for cardiovascular applications has been reported $[6,10-12]$ its interactions with blood have not been carefully characterized $[10,11]$. Therefore, in this study, we assess the hemocompatibility of $\mathrm{BC}$ and of a BC/PVA nanocomposite.

\section{Experimental}

\subsection{Bacterial cellulose and bacterial cellulose/polyvinyl alcohol}

Gluconacetobacter xylinus (ATCC 700178) was grown in a modified Hestrin-Schramm medium, supplemented with $2 \%$ Corn Steep Liquor (Sigma Aldrich, Germany) and 0.6\% ethanol, at pH 5.0. The medium was inoculated and then added to 24-well polystyrene microtitter plates. The plates were then incubated at $30^{\circ} \mathrm{C}$ for 7 days.

The resulting $\mathrm{BC}$ membranes were then washed thoroughly with distilled water and further purified with $4 \% \mathrm{NaOH}$ at $60^{\circ} \mathrm{C}$ for $90 \mathrm{~min}$. The membranes were then, again, thoroughly washed until neutral $\mathrm{pH}$ was achieved.

For BC/PVA production, purified $\mathrm{BC}$ membranes were immersed in a $10 \% \operatorname{PVA}\left(M_{\mathrm{W}}=31,000-50,000 \mathrm{~g} / \mathrm{mol}\right)$ (Sigma Aldrich, Germany) solution for $24 \mathrm{~h}$ at $80^{\circ} \mathrm{C}$. The membranes were then frozen at $-20^{\circ} \mathrm{C}$ for $24 \mathrm{~h}$, after which they were thawed at room temperature in distilled water and washed to remove any polymerized PVA particles on the surface of the membranes.

All membranes were sterilized by washing in $70 \%(\mathrm{v} / \mathrm{v})$ ethanol for $24 \mathrm{~h}$ and then with ultrapure autoclaved water in a vertical laminar air flow hood chamber until ethanol was fully removed.

\subsection{Preparation of blood samples}

Whole blood was collected from healthy donors at Hospital São João (Oporto, Portugal) with citrated (3.2\%) $1.8 \mathrm{ml}$ vacuum blood-collection tubes and transported on ice. The blood samples were then, unless mentioned otherwise, centrifuged at $2000 \times \mathrm{g}$ for 10 min at $4{ }^{\circ} \mathrm{C}$ to obtain Platelet Poor Plasma (PPP), which was then used in the plasma recalcification and Factor XII activation assays.

\subsection{Whole blood clotting times}

Whole blood kinetic clotting times of wet and dried $\mathrm{BC}$ and BC/PVA membranes were determined as previously described by Motlagh [21]. Clotting was induced by addition of $360 \mu \mathrm{l}$ of $0.1 \mathrm{M}$ $\mathrm{CaCl}_{2}$ to $3.6 \mathrm{ml}$ of whole blood. The $\mathrm{BC}$ and $\mathrm{BC} / \mathrm{PVA}$ membranes along with glass microspheres (positive control) and empty wells of a 24-well polystyrene microtitter plate (negative control) were incubated for $0,5,10,15,25$ and $35 \mathrm{~min}$ at room temperature (16-18 ${ }^{\circ} \mathrm{C}$ ) with $150 \mu \mathrm{l}$ of the activated whole blood for each time point. At the end of each time point $3 \mathrm{ml}$ of distilled water was added to the well and incubated for $5 \mathrm{~min}$, in order to lyse the red blood cells which were not trapped in the thrombus, and release hemoglobin.

The concentration of released hemoglobin was measured by transferring $200 \mu \mathrm{l}$ of the supernatant to a 96 -well microtitter plate followed by spectrophotometric analysis at $\lambda=540 \mathrm{~nm}$. The clot formation is followed by a reduction in the absorbance value. All samples, including ePTFE as a control, were analyzed in triplicate.

\subsection{Plasma recalcification profiles}

Plasma recalcification times for BC and BC/PVA membranes, along with empty polystyrene plate wells with (positive control) and without $\mathrm{CaCl}_{2}$ (negative control) were determined by the method described by Motlagh [21]. Samples containing $500 \mu \mathrm{l}$ of Platelet Poor Plasma (PPP) were incubated for $1 \mathrm{~h}$, at $37^{\circ} \mathrm{C}$, on an orbital shaker. After the incubation period, $100 \mu$ l of the PPP from each sample well was transferred to a 96-well plate and $100 \mathrm{ml}$ of $\mathrm{CaCl}_{2}$ was added to each well (except the previously mentioned negative control). The 96-well microtitter plate was then immediately placed in a plate reader, and the kinetics of the clotting measured and monitored by changes in the absorption at $\lambda=405 \mathrm{~nm}$, every $30 \mathrm{~s}$ for $45 \mathrm{~min}$ (curves generally stabilized after $30 \mathrm{~min}$ ). The measure of thrombogenicity was given by the time it took to achieve half maximum absorbance (EC50). Each sample was measured in triplicate ( 3 donors per sample) and averaged; ePTFE was also analyzed in the same way.

\subsection{Hemolysis index}

Hemolysis studies were conducted in accordance with the procedures described by the American Society for Testing and Materials (ASTM F756-00, 2000). BC samples were equilibrated in phosphate buffer saline (PBS) and then transferred to a tube containing $7 \mathrm{ml}$ of PBS. $1 \mathrm{ml}$ of diluted blood (hemoglobin concentration of $10 \mathrm{mg} / \mathrm{ml}$ ) was added and incubated at $37^{\circ} \mathrm{C}$ for $3 \mathrm{~h}$ in a water bath. The tubes were gently inverted every $30 \mathrm{~min}$ to promote contact between blood and samples. The membranes were then removed with sterile tweezers and the diluted blood centrifuged at $750 \times \mathrm{g}$ for $15 \mathrm{~min}$. Then, $1 \mathrm{ml}$ of Drabkin's reagent (Sigma-Aldrich, Germany) was added to $1 \mathrm{ml}$ of supernatant and incubated for $15 \mathrm{~min}$ at room temperature, and finally the absorbance was read at $\lambda=540 \mathrm{~nm}$.

Hemoglobin concentration was calculated using a calibration curve previously prepared with human hemoglobin (Sigma-Aldrich, Germany) and calculated using the formula: $\mathrm{HC}=A \times m \times d$ ( $A$, absorbance; $m$ slope of the hemoglobin curve; $d$, dilution) and presented as percentage. Ultrapure water and PBS served as the positive and negative controls, respectively. ePTFE was analyzed in the same manner as control materials.

\subsection{Complement system activation}

To determine whether the biomaterials activate the complement cascade, we performed the protocol described by the NCL (Nanotechnology Characterization Laboratory) for qualitative 
determination of total complement activation by Western blot analysis (http://ncl.cancer.gov/working_assay-cascade.asp) with slight modifications. Human plasma, from healthy donors, was incubated with $\mathrm{BC}$ and BC/PVA membranes in the presence of veronal buffer. Equal volumes ( $100 \mu \mathrm{l})$ of plasma, buffer and sample were mixed together and incubated at $37^{\circ} \mathrm{C}$ for $60 \mathrm{~min}$. Cobra venom factor (CVF) from Quidel Corporation (San Diego, CA, USA), and PBS were used as positive and negative controls, respectively.

In order to determine if complement proteins could have unspecific adsorption on the BC and BC/PVA membranes, several pre-incubations were performed:

1. Preincubation for $2 \mathrm{~h}$ at $37^{\circ} \mathrm{C}$ with a pool of active plasma followed by a $1 \mathrm{~h}$ incubation with plasma and veronal buffer at $37^{\circ} \mathrm{C}$.

2. Preincubation for $2 \mathrm{~h}$ at $37^{\circ} \mathrm{C}$ with a pool of inactive plasma and then $1 \mathrm{~h}$ incubation with plasma and veronal buffer at $37^{\circ} \mathrm{C}$.

3. Incubation for $1 \mathrm{~h}$ with a pool of plasma and veronal buffer for $1 \mathrm{~h}$ at $37^{\circ} \mathrm{C}$.

4. Incubation of $3 \mathrm{~h}$ with a pool of plasma at $37^{\circ} \mathrm{C}$.

5. Incubation of $3 \mathrm{~h}$ with a pool of plasma and veronal buffer at $37^{\circ} \mathrm{C}$.

Proteins were resolved using 10\% SDS-PAGE, and then transferred to a membrane (Immun-Blot PVDF Membrane, Biorad, Hercules, USA) using the transblot semidry apparatus BioRad transfer equipment (Trans blot SD, BioRad, Hercules, USA). The membranes were incubated for $90 \mathrm{~min}$ with a mouse monoclonal antibody against human C3, diluted to 1:1000 (Abcam, Cambridge, UK). The membranes were then washed and incubated with secondary polyclonal goat anti-mouse IgG antibodies conjugated with alkaline phosphatase, diluted to 1:2000 (Dako, Glostrup, Denmark). The membranes were finally revealed with 5-Bromo-4-Chloro-3Indolyl Phosphate (BCIP) (Sigma Aldrich, Germany).

\subsection{Factor XII activation}

Both free and adhered Factor XII (FXII) activation was determined by a method adapted from Sperling et al. [22]. For the determination of free Factor XII activation BC and BC/PVA membranes were incubated with $300 \mu \mathrm{l}$ of PPP for 1, 5, 10 and 20 min at room temperature $\left(16-18^{\circ} \mathrm{C}\right)$. Then, $30 \mu$ of the plasma was added to $200 \mu$ l of $0.3 \mathrm{M}$ chromogenic substrate S-2302 (Chromogenix, Italy) in $50 \mathrm{mM}$ 4-(2-hydroxyethyl)-1-piperazineethanesulfonic acid buffer (HEPES) and $120 \mathrm{mM} \mathrm{NaCl}$ at $\mathrm{pH} 7.4$; the absorbance was immediately measured at $\lambda=405 \mathrm{~nm}$ for $30 \mathrm{~min}$ (every $30 \mathrm{~s}$ ). An empty polystyrene well and glass microspheres were used as the negative and positive controls. The slope of the resulting curves was calculated and used to compare Factor XII activation. All samples were analyzed in triplicate along with ePTFE.

Additionally, to determine the activation of Factor XII adhered to the surface of BC and BC/PVA, samples incubated with PPP for the same time periods as above $(1,5,10$ and $20 \mathrm{~min})$ were immersed in $300 \mu \mathrm{l}$ of $0.3 \mathrm{M}$ chromogenic substrate S-2302 (chromogenix) in $50 \mathrm{mM}$ HEPES and $120 \mathrm{mM} \mathrm{NaCl}$ at $\mathrm{pH} 7.4$ for $30 \mathrm{~min}$, the reaction was then stopped with $75 \mu \mathrm{l}$ of $20 \%(\mathrm{v} / \mathrm{v})$ acetic acid and $200 \mu \mathrm{l}$ of the reaction volume and the absorbance measured on a 96-well microtitter plate at $\lambda=405 \mathrm{~nm}$.

\subsection{Platelet isolation, characterization and surface activation studies}

Platelet rich plasma (PRP) and purified platelets were obtained by centrifugation of sodium citrate-anticoagulated human blood [23]. Blood collection was organized by the Biobanco Vasco para la Investigación (Basque Biobank for Research, Galdakao, Spain) and performed with informed consent according to the appropriate legal and ethical guidelines. Donors were healthy volunteers without any history of exposure to medication (such as aspirin) or to alcohol in the two weeks prior to blood collection. Purified platelets were suspended in the buffer ( $145 \mathrm{mM} \mathrm{NaCl}, 5 \mathrm{mM}$ Glucose, $1 \mathrm{mM}$ $\mathrm{MgCl}_{2}, 10 \mathrm{mM}$ HEPES, $5 \mathrm{mM} \mathrm{KCl}$, pH 7.4), with (Plt $+\mathrm{Ca}^{2+}$ ) or without $\left(\mathrm{Plt}-\mathrm{Ca}^{2+}\right) 2 \mathrm{mM}$ calcium. Platelet concentration was adjusted to $\approx 1 \times 10^{8}$ cells per $\mathrm{ml}$.

Flow cytometry was used to test platelets for their purity, state of activation in platelet rich plasma (PRP)/purified platelet suspensions and their ability to respond to agonists such as TRAP (thrombin receptor-activating peptide, Sigma-Aldrich, Madrid, Spain) or PMA (phorbol 12-myristate 13-acetate, Sigma-Aldrich, Madrid, Spain). Fluorescently labeled monoclonal antibodies specific for well-known platelet activation markers (CD62P, CD63, active form of GPIIb/IIIa) and fluorescent-annexin 5 (A5) that binds to phosphatidylserine (PS) were used in the flow cytometry experiments. Platelets were identified by staining with anti-CD41a (platelet specific marker). All antibodies and fluorescent markers were purchased from BD Biosciences (Madrid, Spain). Light scatter and fluorescence data from 10,000 events were collected with all the detectors in the logarithmic mode. Flow Jo software (Tree Star Inc, Oregon, USA) was used to determine the percentage of platelets positive for antibody binding for different platelet activation markers. Mean fluorescence intensity for the positive and negative events was determined to obtain the level of expression of various platelet activation markers.

Once platelets were confirmed to be in a quiescent state, as well as responsive to agonists, purified platelets, with and without extracellular $\mathrm{Ca}^{2+}\left(\mathrm{Plt}+\mathrm{Ca}^{2+} ; \mathrm{Plt}-\mathrm{Ca}^{2+}\right)$ or PRP were incubated with clean and sterile BC, BC/PVA and ePTFE membranes. For these experiments, the membranes were cut and deposited into autoclaved eppendorf tube caps. Purified platelets or PRP preparations were incubated with these materials for various time periods ( 10 , 30,50 and $180 \mathrm{~min}$ ) at $37^{\circ} \mathrm{C}$. The entire set up was put inside a clean and covered Petri dish, which was then placed inside a $37^{\circ} \mathrm{C}$ incubator to avoid any contamination. After incubation, the supernatants were removed and collected in an eppendorf tube for immediate analysis by flow cytometry. The non-adherent platelets in the supernatant from each sample were stained for the same markers (CD62P, CD63, PS and activated GPIIb/IIIa) to check for their activation state. The surface adhered platelets were fixed with $2.5 \%$ glutaraldehyde and stained with the antibodies specific for CD41a, CD62P and CD63, for their analysis by confocal microscopy to assess platelet adhesion and activation.

\section{Results and discussion}

\subsection{Whole blood clotting time}

Whole blood was used to determine clotting times and provide information on the thrombogenicity and pro-coagulative activity of each biomaterial. In this assay, whole blood is allowed to clot in contact with a biomaterial. As clotting occurs, more red blood cells are retained in the clot, and therefore less hemoglobin is released by lysis upon addition of distilled water. A hemocompatible material will therefore maintain a higher absorbance value over time. Results show (Fig. 1) that ePTFE and polystyrene (negative control) are the least thrombogenic; conversely, the glass microspheres present the quickest clotting time. BC appears more thrombogenic than either ePTFE or polystyrene, consistent with the results of Andrade et al. [24]. The results for BC/PVA show no statistical difference $(p \geq 0.05)$ compared to those of BC alone. Andrade et al. [24] observed, in agreement with others [25], that whole blood clotting time is related - to some extent - to the surface area of the 


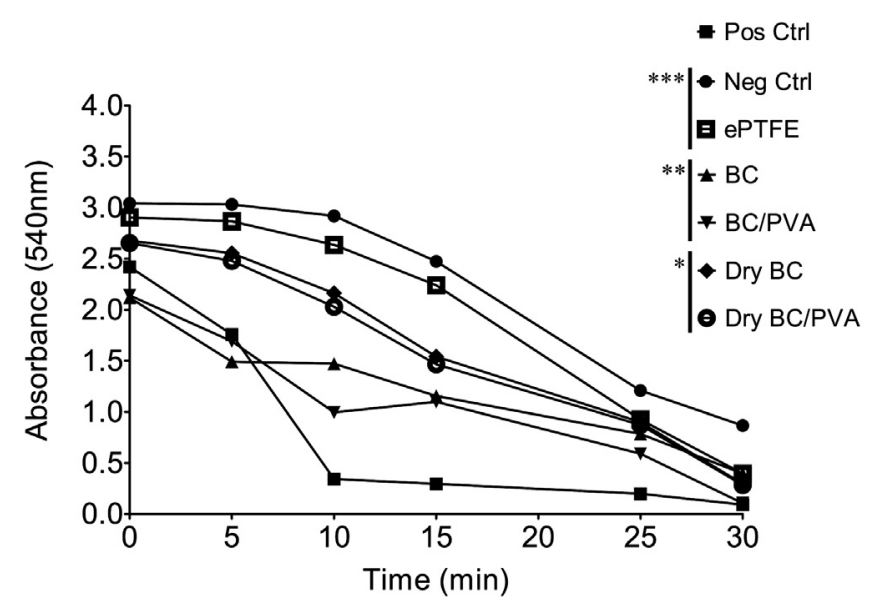

Fig. 1. Whole blood clotting time for wet and dry bacterial cellulose (BC), bacterial cellulose/polyvinyl alcohol (BC/PVA) and expanded polytetrafluoroethylene (ePTFE). The positive $(+)$ control used were glass microspheres and the negative $(-)$ control the polystyrene microtitter plate. Statistically similar data series are marked with alike characters beside the legend. Samples were tested in triplicate.

material. The surface area of the bottom of the microtitter plate well is $1.21 \mathrm{~cm}^{2}$. The ePTFE samples were cut and stretched in order to sit flush and cover the well bottom and so surface of contact was similar to that of the negative control. In the case of both $\mathrm{BC}$ and $\mathrm{BC} / \mathrm{PVA}$, these hydrogels are porous and present a dome shaped structure that does not sit flush to the bottom of the microtitter wells. This increases the surface of biomaterial-blood contact which may adversely affect the comparative analysis of the results. Once dried, BC and BC/PVA membranes lose most of their original thickness (from $3 \mathrm{~mm}$ down to $0.068 \mathrm{~mm}$ in the case of $\mathrm{BC}$ ), hence they are less porous. The resulting dried membranes present a similar surface of contact to that of ePTFE and the negative control. As expected, the clotting time observed on the dried membranes was closer to that of both the negative control and ePTFE. Once again, no significant difference was observed between $\mathrm{BC}$ and $\mathrm{BC} / \mathrm{PVA}$.

\subsection{Plasma recalcification profile}

The plasma recalcification profile obtained in response to PPP contact to a material is often used as a measure of activation of the intrinsic pathway $[21,26]$. As plasma becomes more turbid due to the accumulation of clotting by-products, absorbance increases. The measure of pro-coagulative activity in this test is given by the time it takes to achieve half the maximum absorbance; the higher this value, the less thrombogenic the material. For this assay (Fig. 2) glass microspheres serve as a positive control. The glass microspheres are generally accepted as positive controls in this respect due to their negatively charged surface that triggers protein adsorption and activates coagulation factors.

The positive control was found to be significantly different $(p<0.05)$, with no statistical differences found among the other tested samples. Similarly to what was reported by Andrade [24], ePTFE presents a lower pro-coagulating activity than BC (higher half maximum absorbance time -6.5 versus $5.5 \mathrm{~min}$, respectively). Of particular note is the result for BC/PVA, which outperforms ePTFE. This suggests that the incorporation of PVA improves the performance of $\mathrm{BC}$, as may be inferred from the higher half maximum absorbance time ( $7 \mathrm{~min})$.

\subsection{Factor XII activation}

In order to further characterize the activation of the intrinsic pathway, direct quantification of the catalytic activity of both

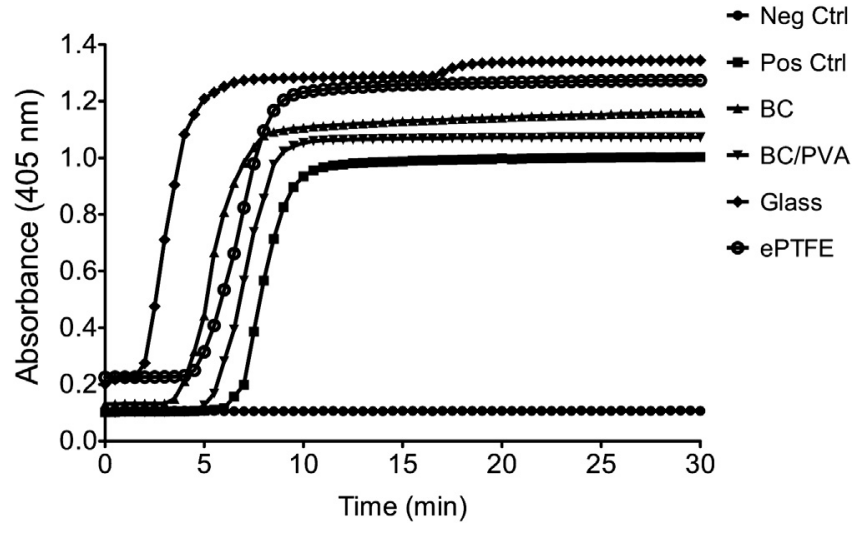

Fig. 2. Plasma recalcification profile of Platelet Poor Plasma (PPP) in the presence of bacterial cellulose (BC), bacterial cellulose/polyvinyl alcohol (BC/PVA) and expanded polytetrafluoroethylene (ePTFE) along with glass microspheres. The positive control was obtained by addition $\mathrm{CaCl}_{2}$ to PPP in a polystyrene plate. Samples were tested in triplicate.

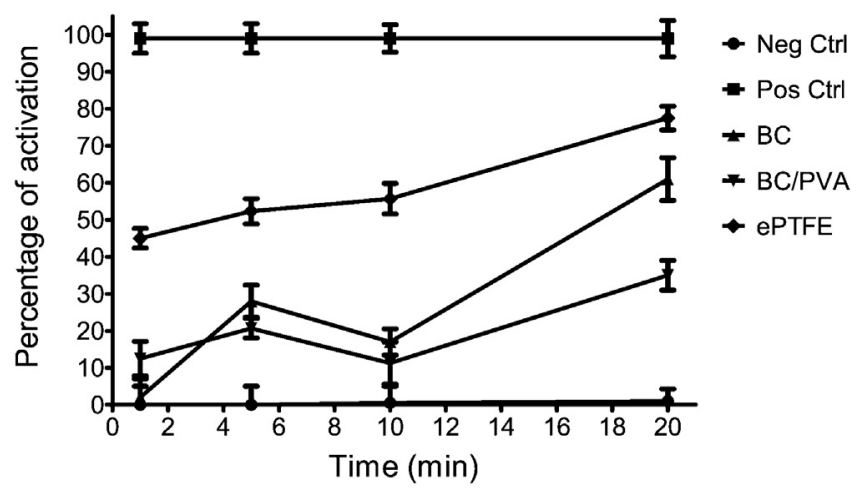

Fig. 3. Percentage of Factor XII Activation, as compared to glass microspheres (+ Control), of bacterial cellulose (BC), bacterial cellulose/polyvinyl alcohol (BC/PVA), expanded polytetrafluoroethylene (ePTFE) and polystyrene (- Control). Measurements were obtained from absorbance of chromogenic substrate S-2302 (chromogenix). Samples were tested in triplicate and presented with standard deviation.

free (Fig. 3) and adhered (Fig. 4) activated Factor XII was performed using a chromogenic substrate. The chromogenic substrate (S-2302) is broken down, specifically, by the activated form of Factor XII (Factor XIIa). The results are expressed as the Factor XII

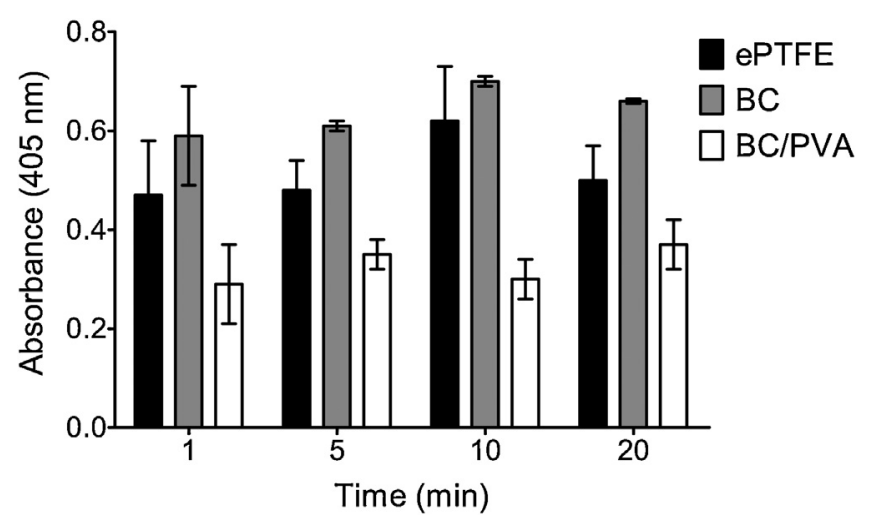

Fig. 4. Absorbance of adhered Factor XII on the surface of bacterial cellulose (BC), bacterial cellulose/polyvinyl alcohol (BC/PVA) and expanded polytetrafluoroethylene (ePTFE). The activity of Factor XII was measured by use of chromogenic substrate S-2302 (chromogenix). Data is presented as mean absorbance with standard deviation $(n=3)$. 
activation upon exposure to the materials, normalized to the value obtained using the positive control (glass microspheres).

The results obtained with $\mathrm{BC}$ and $\mathrm{BC} / \mathrm{PVA}$ present no statistical differences $(p \geq 0.05)$. The thrombogenicity of both materials is however different from that of ePTFE: they induce less free Factor XII activation; the differences are statistically significant (Fig. 3).

All of the materials showed some adhered Factor XIla (Fig. 4). Similar amounts are detected on BC and ePTFE, with no statistical difference between them ( $p \geq 0.05)$. On the other hand, BC/PVA presents significantly $(p<0.05)$ lower values for adhered Factor XIIa than the other two materials. Overall, BC/PVA seems to be triggering the lowest response of the intrinsic coagulation cascade. Thus, again, PVA seems to a have a beneficial impact on the BC hemocompatibility.

It is generally accepted that synthetic materials with a negatively charged surfaces trigger the activation of Factor XII via conformational change to a Factor XIIa-similar molecule $[27,28,30]$. We expect that the surface charges on BC, BC/PVA, and ePTFE are similar and small compared to the highly charged glass spheres. Therefore the differences we observe in the propensity of these materials to activate FXII are not related to differences in their surface charge but to their hydrophilicity/hydrophobicity. Indeed, while some authors suggest that there is little difference in terms of FXII activation between hydrophobic and hydrophilic surfaces [27], others assert that hydrophilic surfaces are more prone to induce Factor XII activation [22]. This is thought to happen due to the socalled adsorption-dilution that occurs on the hydrophobic surfaces, where more proteins adsorb in general and FXII is diluted.[27] We observe the opposite: the more hydrophilic BC and BC/PVA hydrogels are less effective in activating FXII than the hydrophobic ePTFE.

It must be realized, however, that activation of Factor XII does not suffice for the activation of the intrinsic pathway, the complex interactions between several proteins on the surface of the materials may result in more or less effective amplification [31].

\subsection{Platelet adhesion and activation studies}

While platelet adhesion to BC [29] and PVA grafts [32] has already been described in the literature, the detailed characterization of platelet response on these surfaces, especially the newly developed PVA-modified BC surface, has not been done. Here we studied the adhesion and activation of platelets upon interaction with the BC-based materials. To do so, we exposed BC and $\mathrm{BC} / \mathrm{PVA}$ membranes to PRP for different time periods - 10, 30 , 50 and $180 \mathrm{~min}-$ at $37^{\circ} \mathrm{C}$ and assayed for the expression of the activation markers on the surface-adhered platelets and on the platelets in the bulk solution above the surfaces by immunostaining.

We studied four classical markers of platelet activation: transmembrane proteins CD62P (P-selectin) and CD63 (present in the membranes of the granules stored inside the resting platelets and brought to the surface upon activation), active conformation of the integrin complex GPIIb-IIIa, (constitutively expressed on platelet surfaces but undergoes conformation changes upon activation), and phospholipid phosphatidyl serine (located in the cytoplasmic leaflet of the cell membrane in the resting platelets but is brought to the outer leaflet upon activation) [33]. Surface-adhering platelets were studied using confocal laser scanning microscopy (CLSM). The results of these experiments, performed with BC, BC/PVA and ePTFE are shown in Fig. 5. After $10 \mathrm{~min}$, very few platelets adhered on either BC or BC/PVA as compared to ePTFE, which showed a higher level of platelet adhesion (cf. the number of platelets on the various surfaces in Fig. 5A). More platelets were found on $\mathrm{BC}$ and $\mathrm{BC} / \mathrm{PVA}$ surfaces after $50 \mathrm{~min}$ of incubation, while no significant difference in the number of platelets between 10 and 50 min was observed on ePTFE (Fig. 5B); larger patches/clusters could sometimes be observed after 50 min (arrowhead in Fig. 5B). Surface-adhered platelets were found to be activated (expressed CD62P and CD63) on BC and ePTFE surfaces both after 10 and $50 \mathrm{~min}$ (Fig. 5A and B). Judging from the level of fluorescence intensity, the level of expression of CD62P and CD63 was higher on ePTFE than on BC. BC/PVA-adhered platelets were activated only after $50 \mathrm{~min}$ (Fig. 5B). The level of activation in this case was also lower than that observed on ePTFE. Similar results were obtained for purified platelets incubated with surfaces in the absence of calcium (data not shown). Platelet response observed on ePTFE is consistent with the literature where it has been stated that protein adsorption on the ePTFE surface leads to adhesion and activation of platelets [35].

In order to have a better understanding of the interactions between platelets and these materials, we further studied the behavior of platelets in the bulk solution, above the biomaterial surface. Previous studies have shown that in addition to platelets adhering to the material surface, the non-adherent platelets may get activated upon blood-biomaterial interaction [34,35]. These fluid-phase activated platelets contribute to thrombotic and inflammatory reactions at a distance from the original implantation site [36-38]. The activation of non-adherent platelets was investigated using flow cytometry [39]. The results for these experiments are shown in Figs. 6 and 7. We determined the percentage of cells positive for a particular surface marker (Fig. 6) and also the level of expression of that marker (calculated from mean fluorescence intensity) on the positive cells (Fig. 7). The degree of non-adhered platelet activation upon contact with the surface is here presented as an increase in the expression of a particular marker as compared to the freshly isolated resting platelets (Fig. 7, Mean fluorescence intensities ratio, MFIR). Both PRP and purified platelets were used in the experiments. For PRP, CD62P and CD63 expression were used to monitor activation, while for purified platelets all four activation markers (CD62P, CD63, PS and activated GPIIb/IIIa) were used. This is because detection of PS and activated GPIIb/IIIa expression requires extracellular Ca $[40,41]$, which would induce clotting when using PRP.

Freshly isolated platelets were in their quiescent state (Fig. 7) and showed negligible expression of the activation markers until stimulation with TRAP or PMA (positive controls). Following incubation with PRP, no discernible difference in the expression of the activation markers CD62P and CD63 was observed for all 3 biomaterials at time points of 10,30 and $50 \mathrm{~min}$ (Fig. 7A and B). However, a significant difference in the CD62P and CD63 expression was observed after 180 min: ePTFE showed a 13 fold increase in CD62P expression level as compared to $\mathrm{BC}$ and $\mathrm{BC} / \mathrm{PVA}$, which showed a modest 2-3 fold increase relative to the quiescent platelets (Fig. 7A). Similarly, the increase in CD63 expression was 9 fold for ePTFE and 2 fold for BC and BC/PVA (Fig. 7B). Additionally, the results for percentage of cells (Fig. 6) positive for these 2 markers were also in accordance with the trends obtained for their expression levels. For BC and BC/PVA, only $16 \%$ and $18 \%$ of cells, respectively, showed $\mathrm{CD} 62 \mathrm{P}$ expression whereas for ePTFE it was almost much higher (67\%) of BC and BC/PVA (Fig. 6A). Again, for CD63, ePTFE presented much more CD63 positive cells (Fig. 6B) as compared to BC materials ( $80 \%$ and $40 \%$, respectively). These results support our claim that, in the presence of plasma proteins, $\mathrm{BC}$ and $\mathrm{BC} / \mathrm{PVA}$ prove to be more hemocompatible as compared to ePTFE in terms of plasma recalcification profiles, Factor XII activation, platelet adhesion and activation (both in bulk and on surface of the biomaterials).

To complement the results obtained with PRP, we also studied the activation of purified platelets in the presence, as well as in the absence, of calcium. Direct effects of bare surfaces on platelets have 

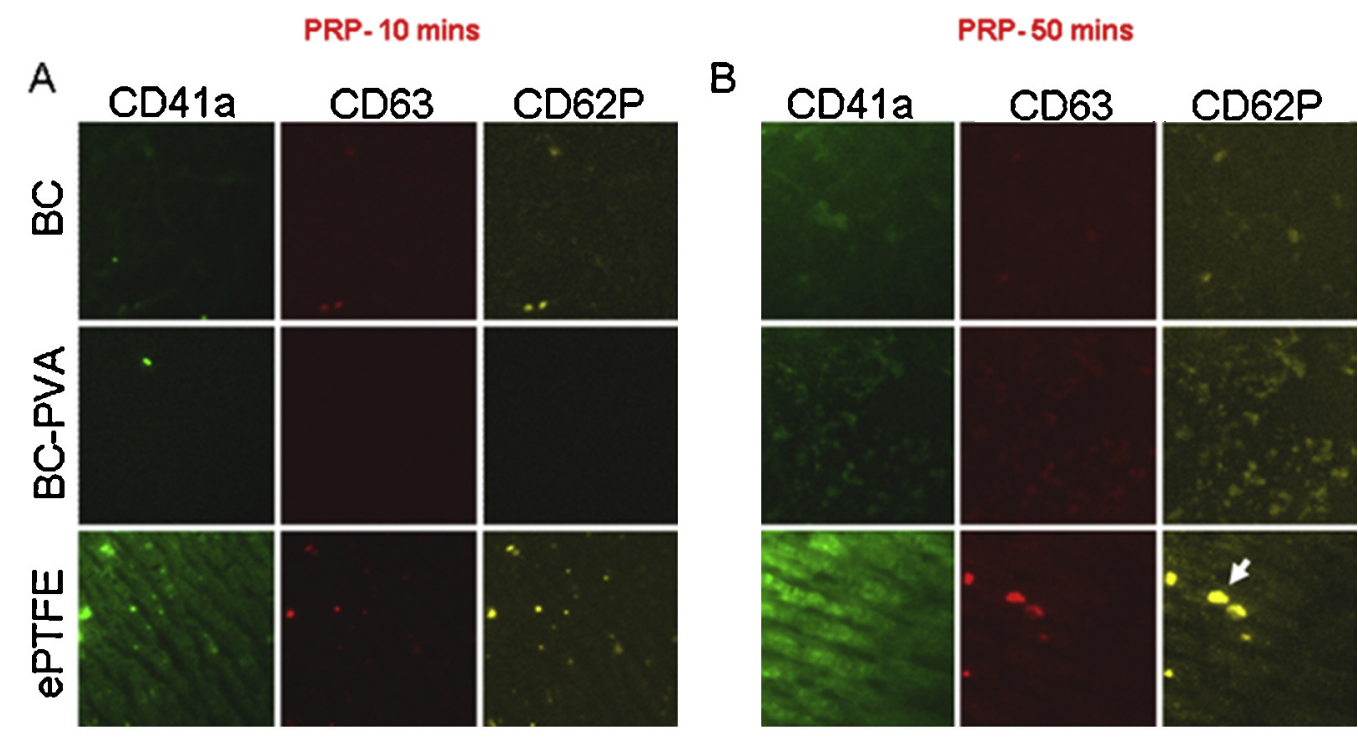

Purified Platelets $+\mathrm{Ca}^{2+}-10$ mins
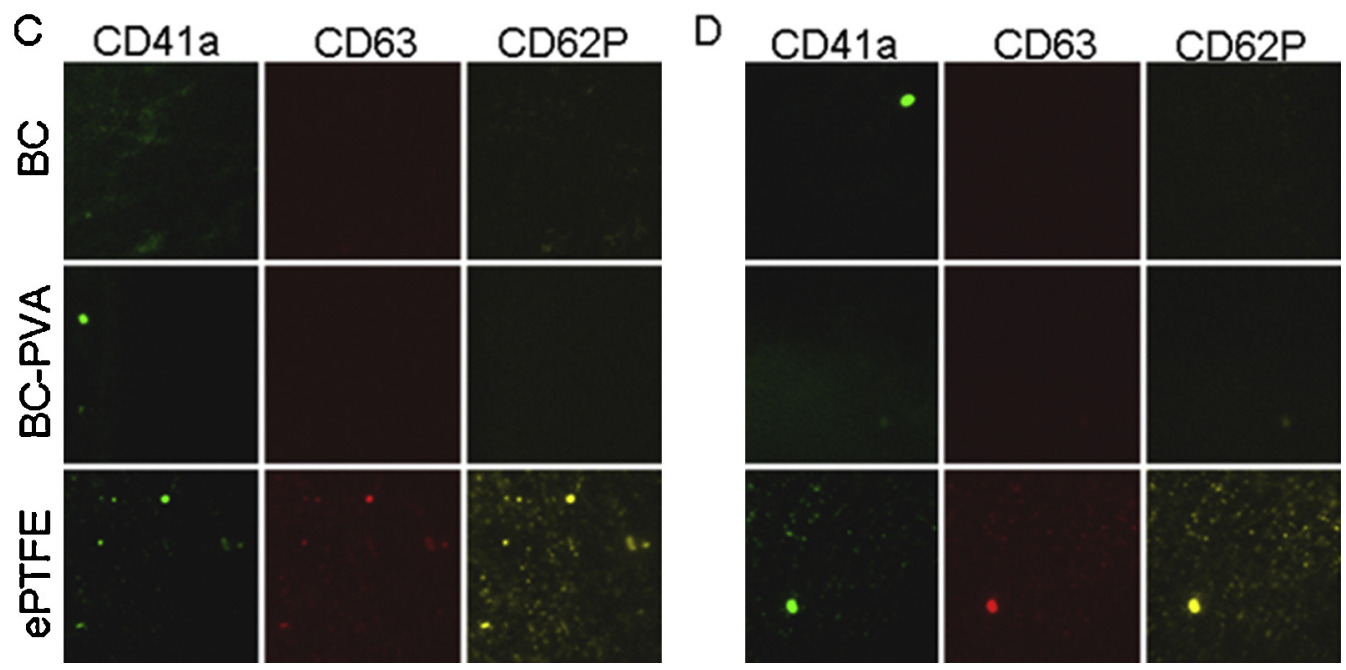

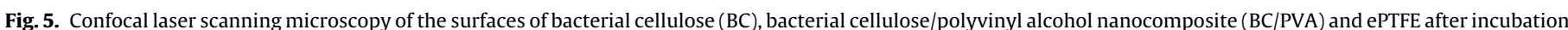

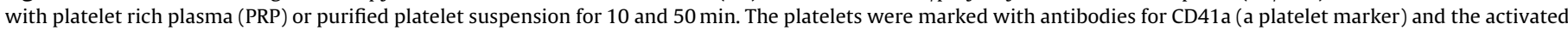
platelet markers CD63 and CD62P.

previously been reported [24,42-46]. The results obtained with surface adhered platelets were already discussed above (Fig. 5), while the results of platelet activation in solution are shown in Figs. 6 and 7 together with those of PRP. The non-adherent platelets incubated with ePTFE showed almost no CD62P/CD63 expression, both in the presence and absence of calcium, for all time points (Fig. 7A and $B$ ). After $180 \mathrm{~min}$, in the absence of calcium, platelets incubated with BC and BC/PVA showed roughly a 2 fold increases in CD62P and $C D 63$ expression, further increased by about 8 and 2 fold, in the presence of calcium, for the same markers. Similarly, BC and BC/PVA presented higher percentage of cells positive for CD62P and CD63 as compared to ePTFE (Fig. 6A and B).

In the case of purified platelets, we could also study the effect of surface interaction on the expression of PS and the active form of GPIIb/IIIa. No increase in the expression of activated form of GPIIb/IIIa relative to that in quiescent platelets was observed for any of the three surfaces (Figs. 6D and 7D). PS expression was found to fluctuate (Fig. 7C). After $180 \mathrm{~min}$, the PS expression is lower (25 fold increase) for BC/PVA as compared to BC (60 fold increase) and ePTFE (50 fold).
The observed PS fluctuations are interesting, but their significance is not altogether clear. They may be related to platelet activation or reflect the loss of PS in the microparticles generated upon activation. The consensus is that PS exposure on the platelet surface is necessary for thrombin generation, but whether it is sufficient for the pro-coagulant function of platelets is still debated [47]. Moreover, there are inconsistencies in literature regarding PS detection, because annexin A5 binding may be interfered with by other PS binding proteins, including the factors of the clotting cascade. Some authors suggest that activation leads to different platelet subpopulations, only some of which expressing PS [48].

In summary, it appears that in the absence of plasma proteins (that is, when using purified platelets), BC and BC/PVA surfaces activate platelets in the bulk more efficiently than ePTFE. Therefore, their superior performance noted in PRP (which better mimic the in vivo conditions) is most likely due to a passivating layer of plasma proteins.

As noted above, the hydrophilic surface of BC and BC/PVA does not favor protein unfolding/conformational changes, hence not leading to Factor XII activation and not causing as much platelet 
A

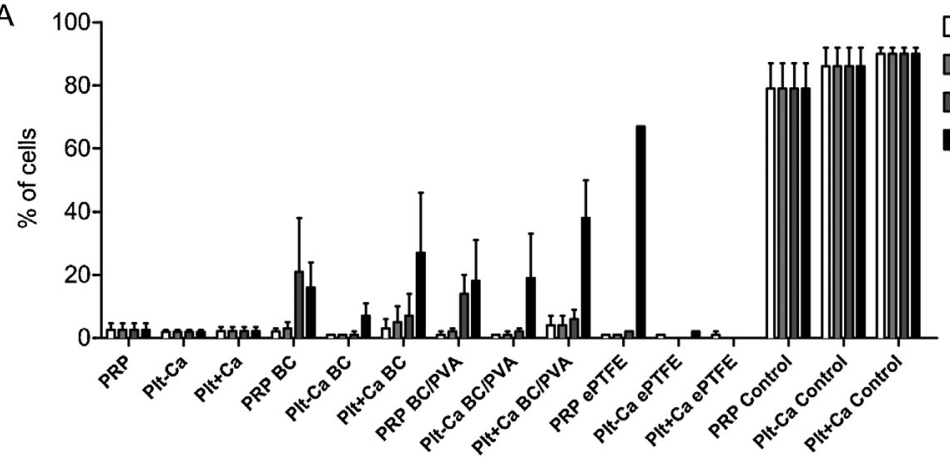

${ }^{8}$

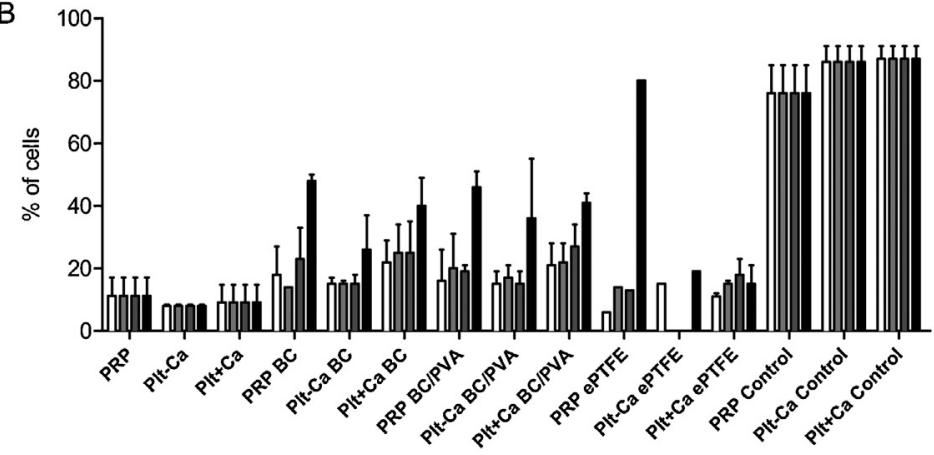

C

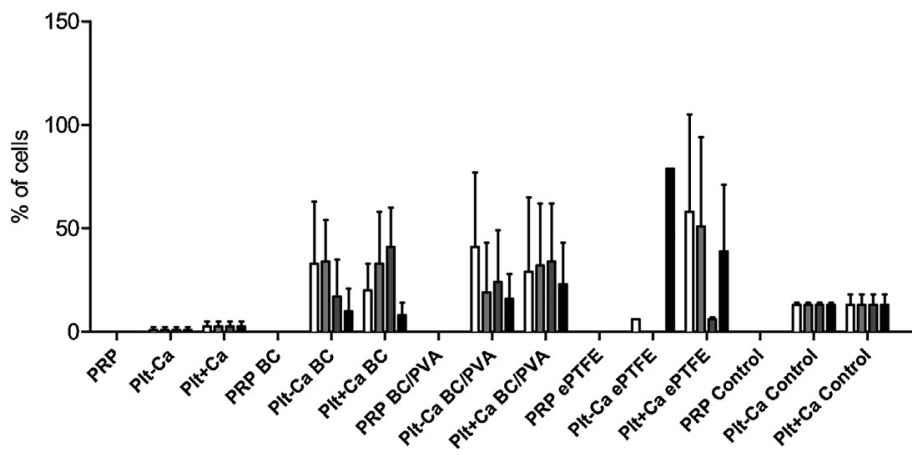

D

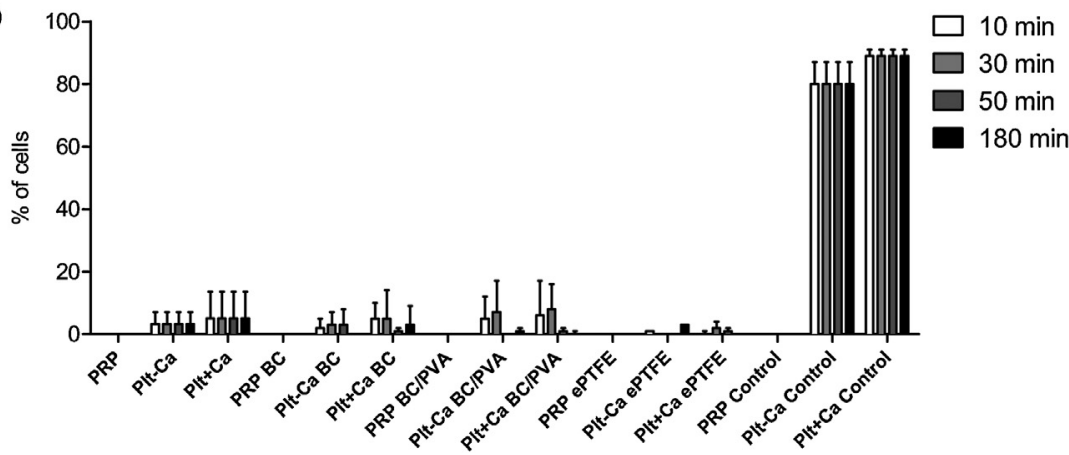

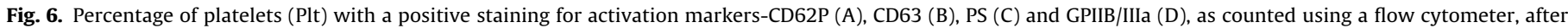

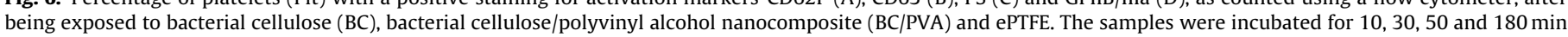

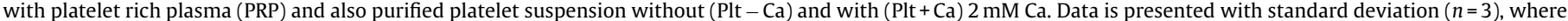
there are no error bars only 2 samples were tested.

activation as protein layers on other surfaces. These results are also in accordance with the findings of Sperling et al. [22] who showed that platelet adhesion and activation was favored by highly hydrophobic surfaces.

\subsection{Hemolytic index}

The hemolytic index is a direct measure of free hemoglobin present in plasma after exposure to a given material or stressor.
An isotonic solution (PBS) served as the negative control and distilled water as the corresponding positive control, inducing osmotic stress that ruptures red blood cells. The assay was performed as according to the Standard Practice for Assessment of Hemolytic Properties of Materials from the American Society for Testing and Materials (ASTM F756-00, 2000); the standard classifies the material as non-hemolytic ( $0-2 \%$ of hemolysis), slightly hemolytic (2-5\% of hemolysis) and hemolytic ( $>5 \%$ of hemolysis). Our results (Table 1 ) show that both $\mathrm{BC}$ and $\mathrm{BC} / \mathrm{PVA}$ are classified, as according to the 

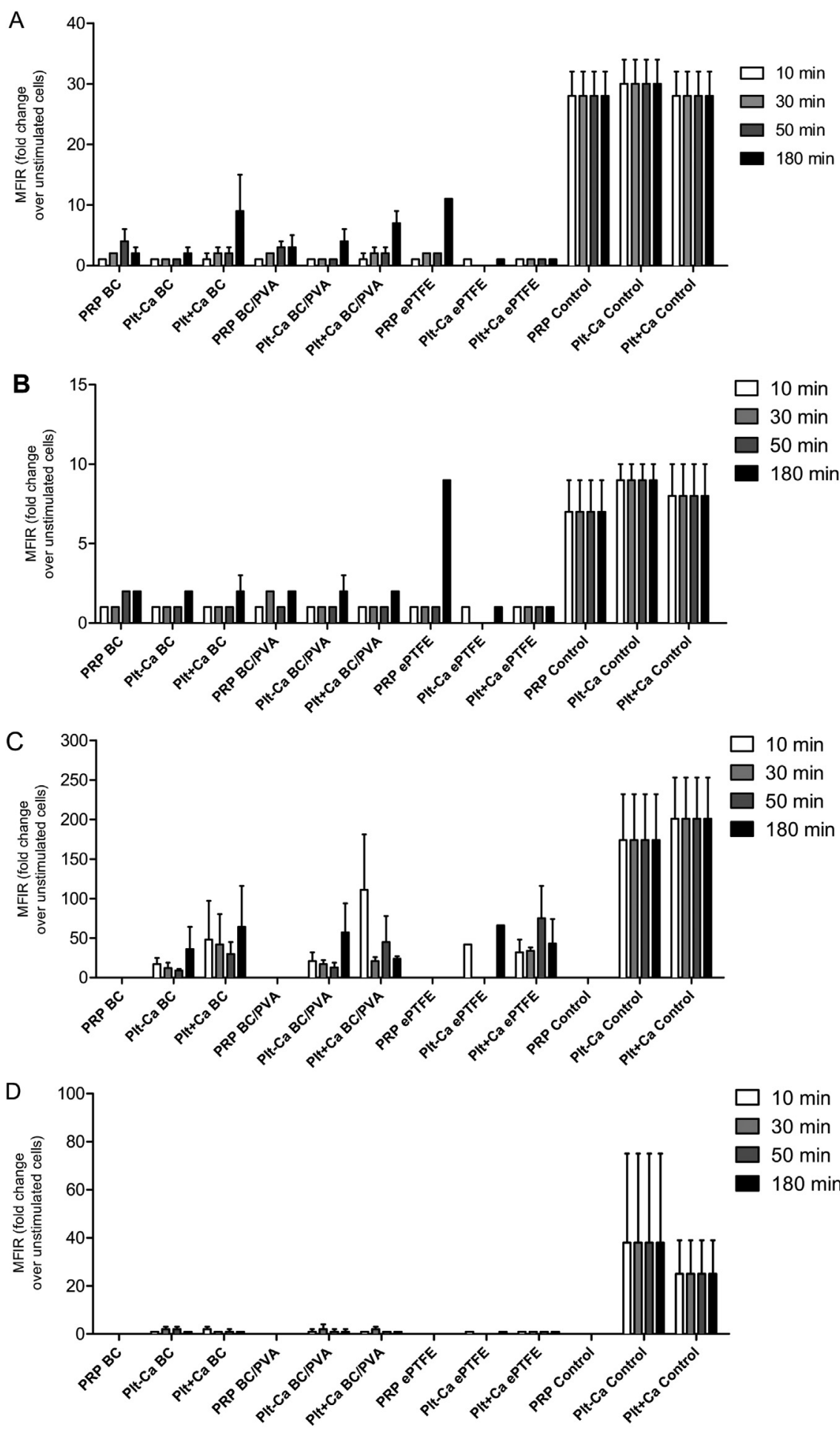

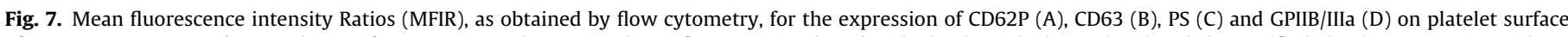

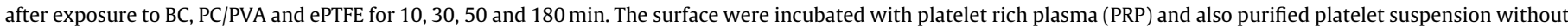
(Plt $-\mathrm{Ca}$ ) and with (Plt $+\mathrm{Ca}) 2 \mathrm{mM} \mathrm{Ca}$. Data is presented with standard deviation $(n=3)$, where there are no error bars only 2 samples were tested.

standard, as non-hemolytic while, ePTFE is classified as slightly hemolytic.

\subsection{Complement system activation}

In order to further study the blood/material interaction, we also looked at the activation of the complement system by $\mathrm{BC}$ and $\mathrm{BC} / \mathrm{PVA}$. Among the three possible mechanisms the alternative activation pathway is of particular importance. In blood/biomaterial interactions the alternative activation pathway is triggered directly by foreign surfaces that do not provide adequate down-regulation of the protease C3-convertase.

We determined the overall percentage of C3 cleavage products, by semi-quantitative Western blot analysis, in order to determine the degree of activation of the complement system. The results are presented as the percentage of cleaved products as compared to 


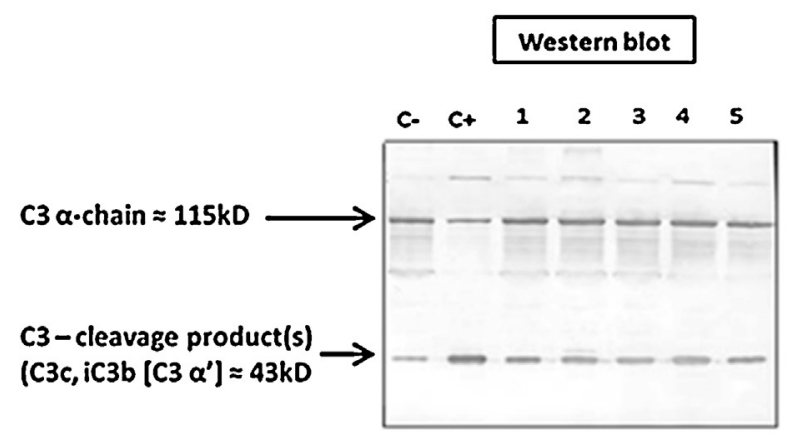

Percentage of $\mathrm{C3}$ cleavage
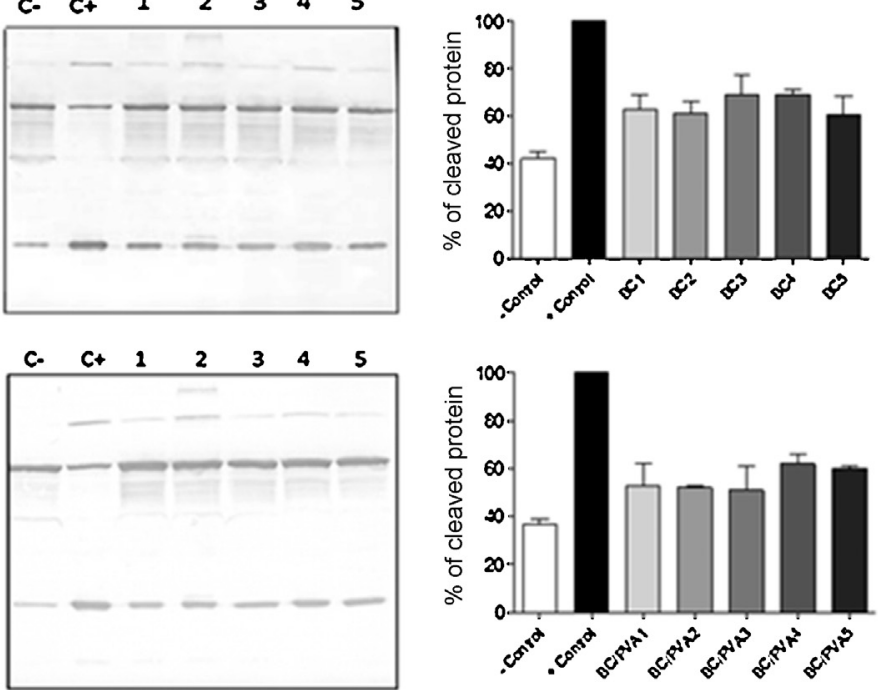

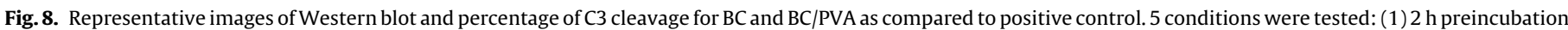

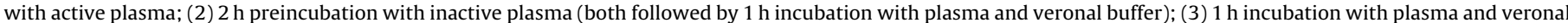

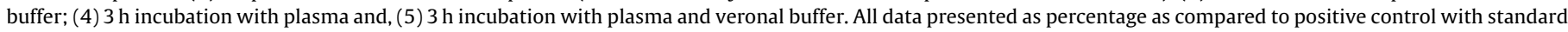
deviations $(n=3)$ no statistical differences between tested conditions $(p \geq 0.05)$.

the positive control, obtained by addition of cobra venom factor to the plasma. Our results show that both $\mathrm{BC}$ and BC/PVA significantly activate complement (Fig. 8). Complement activation of the $\mathrm{BC}$ samples presents an average of $64.5 \pm 4.2 \%$. The effect is slightly mitigated in the case of BC/PVA with an average of $55.6 \pm 5.0 \%$ activation. Considering the negative control presents an average $39.6 \pm 4.0 \%$ this means an increase of $24.9 \%$ for $\mathrm{BC}$ and $16.0 \%$ for BC/PVA. Additionally, dry BC was also tested (results not shown) and was found not to activate the complement system, the values obtained were lower than those of the negative control.

The mechanisms by which complement is activated are still not fully understood but it is well established that different biomaterial surfaces have different complement-activating properties. Physical properties such as hydrophobicity affect the activating ability. Hydrophobic surfaces are more potent activators than hydrophilic ones, and incorporation of chemical groups such as $-\mathrm{NH}_{2},-\mathrm{OH}$ or $-\mathrm{COOH}$ influences the activation of complement [49]. Both BC and $\mathrm{BC} / \mathrm{PVA}$ have many surface - $\mathrm{OH}$ groups which would in turn activate the complement system to some extent. However, the total increase of C3 cleavage products is relatively low, as compared to the negative control.

Interestingly, in both the presence of PVA and in the dry BC membranes, complement system activation is mitigated or canceled out. This may be due to a probable reduction of the total number of free surface $-\mathrm{OH}$ groups, resulting in reduced biomaterial derived C3 cleavage [49].

Table 1

Blood hemolysis index after contact with of bacterial cellulose (BC), bacterial cellulose/polyvinyl alcohol (BC/PVA) and expanded polytetrafluoroethylene (ePTFE).

\begin{tabular}{lr}
\hline Samples & $(\%)$ \\
\hline PBS & 1.84 \\
$\mathrm{mQ} \mathrm{H}_{2} \mathrm{O}(\mathrm{C}+)$ & 95.34 \\
$\mathrm{BC}$ & 1.85 \\
$\mathrm{BC} / \mathrm{PVA}$ & 1.94 \\
ePTFE & 2.42 \\
\hline
\end{tabular}

\section{Conclusions}

BC and PVA have been extensively studied by various research groups for biomedical applications in blood contacting materials. This is the first study, to our knowledge, to present extensive results on the hemocompatibility of a nanocomposite of $\mathrm{BC}$ impregnated with PVA, namely in regards to platelet activation profiles on $B C$ and the $\mathrm{BC} / \mathrm{PVA}$ nanocomposite. The results consistently show that these materials has a good hemocompatibility, probably due to both low activation of platelets and Factor XII.

The results for platelet adhesion and activation profiles demonstrate that both $\mathrm{BC}$ and $\mathrm{BC} / \mathrm{PVA}$ exhibit superior performance as compared to ePTFE. Platelet activation on ePTFE seems to be mediated by plasma proteins. It appears that, on ePTFE, activation of the intrinsic pathway (higher Factor XII activation, Fig. 3) leads to higher thrombin generation and this could explain the high level of platelet activation observed on this surface. The indication that platelets can be activated by $\mathrm{BC}$ and $\mathrm{BC} / \mathrm{PVA}$ is relevant; however, it seems to be mitigated by the presence of other plasma constituents. $\mathrm{BC}$ appears thus as a suitable material for the development of cardiovascular grafts.

\section{Acknowledgments}

We would like to acknowledge Dr. África González Fernández of the Department of Biochemistry, Genetics and Immunology of the University of Vigo, Spain; for providing the know-how and logistics for the complement system activation analysis and, Dra. Manuela Carvalho of the Center of Thrombosis, Hemostasis and Vascular Biology, Department of Blood Transfusion and Blood Bank, Hospital São João, Oporto, Portugal for organizing the blood collection in Portugal.

We would also like to acknowledge Drs. Laura Saa and Valeri Pavlov allowing us to use their centrifuge; Drs. Jose Manuel Cardenas Diaz De Espada, Roberto Bilbao Urquiola, Amaia del Villar Álvarez, and Ms. Clara Rodriuez Aierbe (Basque Biobank for Research, Galdakao, Spain); Ms. Mari Carmen Pozo and Dr. Mari 
Mar Lertxundi (Basque Biobank for Research, San Sebastian, Spain) for organizing the blood collection and testing in Spain.

Funding for this work was made possible by the FCT funding through the project PTDC/EBB-EBI/112170/2009, FCT PhD grant (SFRH/BD/66094/2009) and COST-Short Term Mission (COSTSTSM-TD1003-8978). Additionally, funding from the Department of Industry of the Basque Government program ETORTEK and from the Spanish Ministry of Science and Innovation (basic research grant CTQ2009-11245 to IR) are also gratefully acknowledged.

\section{References}

[1] J. Chulpác, E.L. Filová, Bačákov. Physiol. Res. 58 (2009) S119-S139.

[2] W.B. Tsai, J.M. Grunkemeier, T.A. Horbett, J. Biomed. Mater. Res. 44 (1999) 130-139.

[3] S. Schopka, T. Schmid, C. Schmid, K. Lehle, Materials 3 (2010) 638-655.

[4] R.W. Colman, A.H. Schmaier, Blood 90 (1997) 3819-3843.

[5] Y.J. van der Zijpp, A.A. Poot, J. Feijen, Arch. Physiol. Biochem. 111 (2003) 415-427.

[6] D. Klemm, D. Schumann, U. Udhardt, S. Marsch, Prog. Polym. Sci. 26 (2001) 1561-1603.

[7] A. Svensson, E. Nicklasson, T. Harrah, B. Panilaitis, D.L. Kaplan, M. Brittberg, P. Gatenholm, Biomaterials 26 (2005) 419-431.

[8] O.M. Alvarez, M. Patel, J. Booker, L. Markowitz, Wounds 16 (2004) 224-233.

[9] J.D. Fontana, A.M. Desouza, C.K. Fontana, I.L. Torriani, J.C. Moreschi, B.J. Gallotti, S.J. de Souza, G.P. Narcisco, J.A. Bichara, L.F. Farah, Appl. Biochem. Biotechnol. 24 (1990) 253-264.

[10] J. Wippermann, D. Schumann, D. Klemm, H. Kosmehl, S. Salehi-Gelani, T. Wahlers, Eur. J. Vasc. Endovasc. Surg. 37 (2009) 592-596.

[11] F.K. Andrade, R. Costa, L. Domingues, R. Soares, M. Gama, Acta Biomater. 6 (2010) 4034-4041.

[12] H. Bäckdahl, B. Risberg, P. Gatenholm, Mater. Sci. Eng. 31 (2010) 14-21.

[13] F.K. Andrade, S.M. Moreira, L. Domingues, F.M. Gama, J. Biomed. Mater. Res. A 92 (2010) 9-17.

[14] F.K. Andrade, R.A.N. Pertile, F. Dourado, F.M. Gama, Bacterial Cellulose: Properties, Production and Applications, Nova science Publishers, 2010.

[15] H. Bäckdahl, G. Helenius, A. Bodin, U. Nannmark, B.R. Johansson, B. Risberg, P. Gatenholm, Biomaterials 27 (2006) 2141-2149.

[16] L.E. Millon, W.K. Wan, J. Biomed. Mater. Res. B: Appl. Biomater. 79B (2006) 245-253.

[17] C. Hassan, P. Peppas, Adv. Polym. Sci. 153 (2000) 37-65.

[18] Y. Mori, H. Tokura, M. Yoshikawa, J. Mater. Sci. 32 (1997) 491-496.

[19] K.C. Chu, B.K. Rutt, Magn. Reson. Med. 37 (1997) 314-319.

[20] A.F. Leitão, J.P. Silva, F. Dourado, M. Gama, Materials 6 (2013) 1956-1966.
[21] D. Motlagh, J. Yang, K.Y. Lui, A.R. Webb, G.A. Ameer, Biomaterials 27 (2006) 4315-4324.

[22] C. Sperling, M. Fischer, M.F. Maitz, C. Werner, Biomaterials 30 (2009) 4447-4456.

[23] S. Gupta, I. Reviakine, Biointerphases 7 (2012) 24

[24] F.K. Andrade, J.P. Silva, M. Carvalho, E.M. Castanheira, R. Soares, M. Gama, J Biomed. Mater. Res. A 98 (2011) 554-566.

[25] D.L. Stewart, T.T. Nguyen, C.B. Beal, 1985, WO/1985/004426.

[26] L. Vroman, Bull. N. Y. Acad. Med. 64 (1988) 352-357.

[27] R. Zhuo, C.A. Siedlecki, E.A. Vogler, Biomaterials 27 (2006) 4325-4332.

[28] X. Chen, J. Wang, Z. Paszti, F. Wang, J.N. Schrauben, V.V. Tarabara, A.H. Schmaier, Z. Chen, Anal. Bioanal. Chem. 388 (2007) 65-72.

[29] H. Fink, J. Hong, K. Drotz, B. Risberg, J. Sanchez, A. Selborn, J. Biomed. Mater. Res. A 97A (2011) 52-58.

[30] H. Fink, L. Faxälv, G.F. Mólnar, K. Drotz, B. Risberg, T.L. Lindahl, A. Sellborn, Acta Biomater. 6 (2011) 1130-1152.

[31] J.H. Park, Y.H. Bae, Biomaterials 23 (2002) 1797-1808.

[32] G. Jin, Q.Z. Yao, L.Q. Dung, F. Ma, Chem. Res. Chin. Univ. 27 (2011) 10781082.

[33] L.K. Jennings, Thromb. Haemost. 102 (2009) 248-257.

[34] S.W. Wang, A. Sen Gupta, S. Sagnella, P.M. Barendt, K. Kottke-Marchant, R.E. Marchant, J. Biomater. Sci. Polym. Ed. 20 (2009) 619-635.

[35] C.H. Gemmell, J. Biomater. Sci. Polym. Ed. 12 (2009) 933-943.

[36] Q. He, K. Gong, Q. Ao, T. Ma, Y. Yan, Y. Gong, X. Zhang, Biomater. Appl. J. (2011) 29.

[37] B.D. Ratner, S.J. Bryant, Annu. Rev. Biomed. Eng. 6 (2004) 41-75.

[38] B.D. Ratner, J. Biomater. Sci. Polym. Ed. 11 (2000) 1107-1119.

[39] B.D. Ratner, The catastrophe revisited: blood compatibility in the 21 st century, Biomaterials 28 (2007) 5144-5147.

[40] S.J. Shattil, M. Cunningham, J.A. Hoxie, Blood 70 (1987) 307-315.

[41] S.J. Shattil, J.A. Hoxie, M. Cunningham, L.F. Brass, J. Biol. Chem. 260 (1985) 11107-11114.

[42] V. Gerke, S.E. Moss, Physiol. Rev. 82 (2002) 331-371.

[43] S.L. Goodman, S.L. Cooper, R.M. Albrecht, J. Biomed. Mater. Res. 27 (1993) 683-695.

[44] B.S. Smith, S. Yoriya, L. Grissom, C.A. Grimes, K.C. Popat, J. Biomed. Mater. Res. A 95 (2010) 350-360.

[45] Y.B. Aldenhoff, L.H. Koole, Eur. Cells Mater. 5 (2003) 61-67.

[46] N. Nurdin, P. François, Y. Mugnier, J. Krumeich, M. Moret, B.-O. Aronsson, P. Descouts, Eur. Cells Mater. 5 (2003) 17-26.

[47] I.C. Munnix, M.J. Kuijpers, J. Auger, C.M. Thomassen, P. Panizzi, M.A. van Zandvoort, J. Rosing, P.E. Bock, S.P. Watson, J.W. Heemskerk, Arterioscler. Thromb. Vasc. Biol. 27 (2007) 2484-2490.

[48] J.W. Heemskerk, N.J. Mattheij, J.M. Cosemans, J. Thromb. Haemost. 11 (2013) $2-16$.

[49] K.N. Ekdahl, B. Nilsson, C.G. Golander, H. Elwing, B. Lassen, U.R. Nilsson, J. Colloid Interface Sci. 158 (1993) 121-128. 